\title{
Cost-effective and Green Manufacturing Substrate Integrated Waveguide (SIW) BPF for Wireless Sensor Network Applications
}

\author{
Hiba Abdel Ali, Rachida Bedira, Hichem Trabelsi, Ali Gharsallah \\ UR of Circuits and Elec. Sys. HF, Dept. of Physics, El Manar University, Faculty of Sciences \\ Tunis, Tunisia
}

\begin{abstract}
This paper presents a comparison between innovative technique for implementation of substrate integrated waveguide band pass filter centered at $4 \mathrm{GHz}$ and conventional PCB results . Two poles filter is designed, simulated and fabricated. The novel fabrication process technique is based on green manufacturing where physical etching of metal layer of aluminum is glued on paper substrate. The pass band filter is composed of two resonant adjacent and symmetric cavities separated by a coupling iris. The same topology is adopted with conventional substrate and PCB technology to validate the new technique results. The bandwidth achieved is almost four times wider than found with PCB technology developed for UWB applications. The proposed technique keeps the advantages of conventional SIW technology including low profile, compact size, complete shielding, easy fabrication, low cost for mass production as well as convenient integration with planar waveguide components including transitions. Moreover, the flexible quality of paper offer the possibility to fabricate conformal shapes of SIW components which is not possible with conventional rigid substrates made with PCB technology. In addition to the advantages of eco friendly, renewable, light weight, and ultra low cost materials.
\end{abstract}

Keywords-Substrate Integrated Waveguide; Band pass filter; Wireless sensor network; green material technology; paper substrate

\section{INTRODUCTION}

During the last two decades, the main issue for microwave and millimeter researchers is to decrease the pollution impact of electronic devices and integrated circuits made with chemical etching process as the conventional PCB and LTCC technologies. Indeed, The major part of these concerns is devoted to develop new green electronic technologies based on eco-friendly materials and ecological process of fabrication. Thus employing new class of microwave clean components. This aim will be achieved during manufacturing, operational life and even after disposal.

In order to satisfy the Industrial and Consumers requirements in microwave devices, tests are multiplied to validate the feasibility of new process of fabrication including materials. In fact, Internet of things and the new generation of wireless sensor networks applications require a large number of sensor node. Thus, using a low cost materials and easy to manufacture process is a necessity.
The proposed manufacturing technique involves physical etching of aluminum foils to design metal shape using milling machine. Chemical etching is avoided together with the use of acids and other high-polluting solutions. Hence, this process can be considered as a solution to reduce the environmental impact in the future of electronic circuits fabrication and paves the way to develop and manufacture green electronic devices for wireless communication systems.

Furthermore, paper and aluminum are two materials very cheap, low weight and commercially available. In addition, these materials are completely recyclable.

Paper is already used in the literature in [1,2], where three layers of photographic paper are glued, then the metal layer is made by dropping the ink with silver nano particles using inkjet printer. This manufacturing process achieve high accuracy but it is so expensive due to the high cost of silver . In addition ,many layer of ink are required to achieve the right thickness of metal layer which becomes very time consuming .Other ecologic substrate used in last few years is textile for wearable applications, such as tracking position of fire fighting men [3].Also, Plastic was exploited for high frequency applications [4]. In [5], process of fabrication has complicated steps and demanding many tools such as UV light source, photo-lithographic etching with photo resist film deposed on the cooper surface and $\mathrm{NaOH}$ solution was used to remove the unimpressed film. Also, protective and sacrificial layer are required. Moreover, the adhesive layer seems a problem with milling machine because it becomes not solid after heating process .

These Investigations are applied for ambient monitoring of gas, temperature, humidity in library, museums and artworks, wall and buildings, hospitals and operating theaters, supply chain sensing and logistics of food industries, agriculture and farming maturation of fruit and vegetables, growth of plants, temperature of green houses. Especially, the light weight and ecologic properties adopted in this work make it suitable to be integrated in a sensor mounted directly on plants.

Moreover, this work covers the Substrate Integrated Waveguide technology. This solution, among different technologies, is particularly suitable for implementing microwave structures: it allows for planar integration of active and passive components as well as antennas, to realize 
systems on ship integrated circuits. Also, it exhibits low loss and complete shielding. Thus, preventing interference and cross-talk phenomena. In addition SIW technology is a mature technology; in fact it has shown incredible advances over the last decade, where a variety of components and systems have been proposed: selective filters, compact and broadband transmission lines, antenna arrays, and active antennas, tunable devices have been designed and tested [10-15] . SIW structure is composed by two rows of metalized vias arranged with a calculated distance to avoid loss radiation even for high frequency and used to realize metal edge walls. Those vias are integrated in a dielectric filled waveguide and spaced width depends on the operation frequency as the traditional rectangular waveguide but in planar form [16-18]. It works in TE or TM modes, instead of the quasi-TEM mode of a microstrip line. Planar transitions such as coplanar waveguides (CPWs) and microstrip lines can be perfectly integrated to SIWs cavities [6-8].

This paper will exploit the advantages of SIW technology mentioned above and depicts the green manufacturing process as well as the reliability of eco friendly material based paper substrate compared to PCB technology.

This paper is structured into four sections: section II shows the manufacturing process, section III displays the proposed prototypes of filter realized based Taconic TLX substrate then with novel technique based paper substrate and section IV concludes the paper.

\section{FABRICATION PROCESS}

The structure is mono-substrate employing single sheet of paper with a thickness of $0.5 \mathrm{~mm}$ which is similar thickness with respect to three layers of Kodak in [1,2]. In fact, air bubbles between the paper sheets are avoided and no more stick-glue is employed to attach three layer of paper, thus, decreasing losses in dielectric comparing to[1,2]. One can say that, this manufacturing process is more reliable than that used in $[1,2]$.
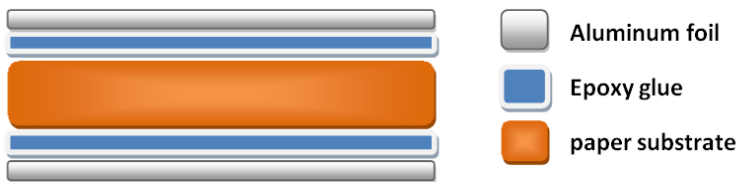

Fig. 1. First step: gluing paper with Aluminum foils

The metallization of paper substrate is simply consisting in gluing both face with aluminum foil using a very thin layer of glue which has no effect on performance of the filter based paper substrate Fig. 1.

The well known aluminium foil of the kitchen as metal layer replacing adhesive cooper used in [5]. To design an SIW microwave component, electromagnetic paper characterization is mandatory. In fact, single sheet of paper with a thickness of $0.5 \mathrm{~mm}$ and a negligible roughness is composed by $50 \%$ of cellulose and $50 \%$ of cotton. The returned electric properties are dielectric permittivity of 2.25 and loss tangent of 0.045 based on a ring resonator over the UWB frequency band. The aluminum foil characterization is required to estimate the total losses of the structure. The aluminum conductivity was characterized at $2.45 \mathrm{GHz}$ and its conductivity is comparable to the standard bulky aluminum [14]. This result shows that aluminum is more conductive than conductive ink for paper ink-jet printing technology used in [1] and the conductive fabrics employed for textile substrate in [4]. At the same time, the use of bi-component epoxy glue represents a very small percentage (in terms of weight) of nonecological materials over the entire structure. In fact, after gluing paper and aluminum, a heating process is required to solidify the glue. This step is very important to realize the device on paper substrate with a computer numerical controlled (CNC) milling machine exactly as conventional PCB technology with SIW Taconic traditional substrate .Subsequently, arbitrary planar shapes are possible on paper substrates like standard one. The tool available spans from the $0.25 \mathrm{~mm}$ in diameter for the circuit edges to the $3 \mathrm{~mm}$ tool for etching wide areas of aluminum. In addition, the milling machine equipped with spiral drill tool can also drilled tens of via holes per minute required to manufacture SIW based components. Once the device is prototyped with the milling machine, the SIW via holes have to be metalized. In order to avoid the spread of the conductive paste within the paper caused by the porosity characteristic of paper, a developed solution is adopted after experimental tests: covering the inner wall of each post with thin layer of epoxy glue. Another fast heating process is required to solidify the coating. Then, after shielding the vias from the effect of spreading issue, a liquid conductive paste is employed on the inner wall of each via to ensure the best ohmic contact between top and bottom aluminum foils. The last heating process in a thermal oven is necessary to increase the conductivity of the conductive paste and therefore the electrical performance of the metallization. The process is simple and easy comparing to [5].

From this work and by means of this new manufacturing process, the costs and complexity of overall structure and systems on chip are significantly decreased $[13,15]$.

\section{EXPERIMENTAL VERIFICATION OF THE FILER PERFORMANCE}

\section{A. SIW Band pass filter with conventional PCB}

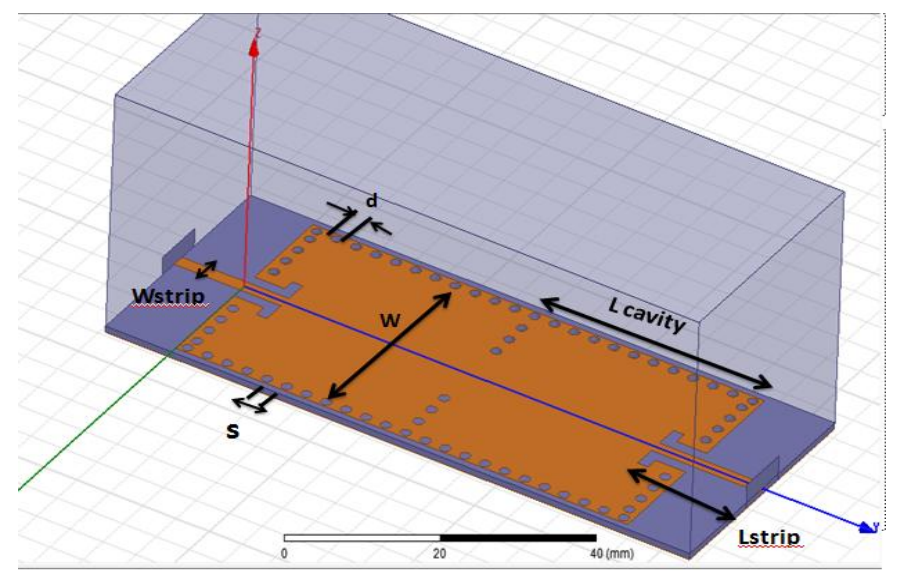

Fig. 2. Geometry of the SIW filter on TLX substrate $(\mathrm{d}=1.5 \mathrm{~mm}, \mathrm{~s}=3 \mathrm{~mm}$, Lstrip $=16.15 \mathrm{~mm}, \quad$ Wstrip $=1.6 \mathrm{~mm}, \quad \mathrm{a}=11.5 \mathrm{~mm}, \quad$ Lcavity $=32.8 \mathrm{~mm}$, $\mathrm{w}=33.7 \mathrm{~mm}$ ) 
Two pole band pass filter is designed simulated and then fabricated. The electric properties of standard substrate Taconic TLX 9 are a relative dielectric permittivity equal to 2.5 and loss tangent of 0.0019 . The height used for this prototype is equal to $0.76 \mathrm{~mm}$. The filter is designed to work on the UWB frequency band. The dimensions of the symmetrical cavities and the iris of coupling between them are optimized for the central operation frequency of $4 \mathrm{GHz}$.

The top layer and geometry of the filter are presented in Fig. 2 while the bottom layer is a fully metalized ground plane.

For the sake of clarity, Fig.3 shows 3D view of the horizontal and vertical symmetry plane of the filter designed in this work. The simulations are carried out with ANSYS HFSS software.

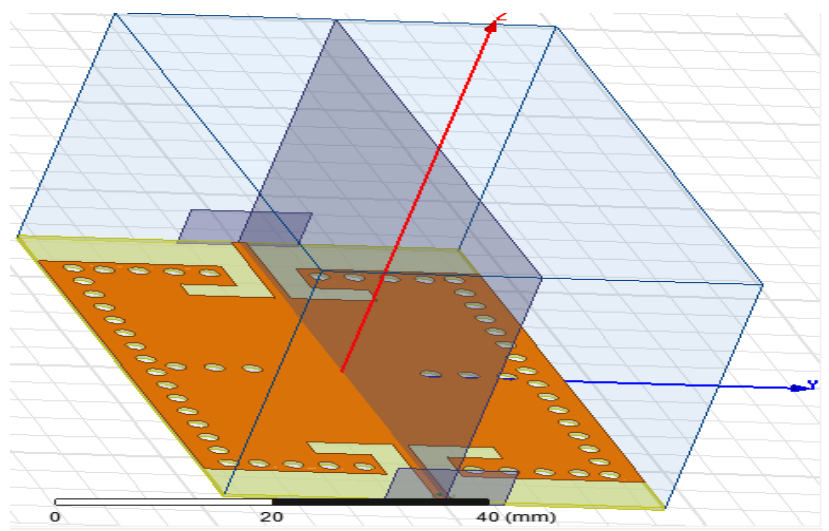

Fig. 3. $3 \mathrm{D}$ topology of the proposed pass band filter with symmetrical plane in HFSS

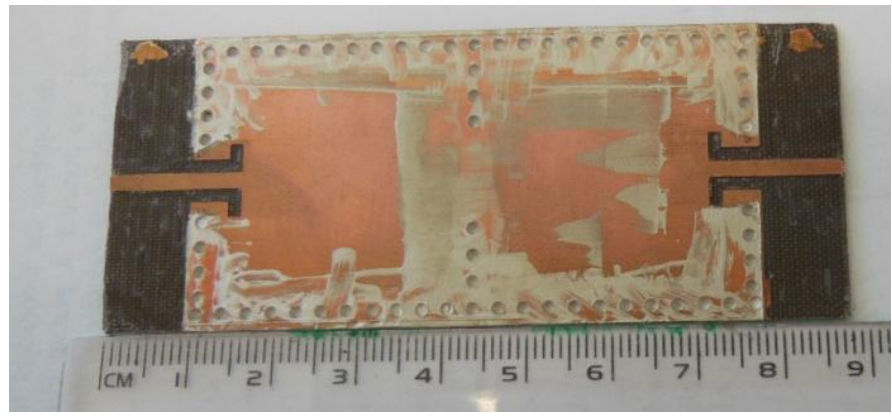

Fig. 4. Top view of fabricated TLX-based SIW filter

Fig. 4 shows the top face of the fabricated TLX substrate filter where vias are metalized with conductive paste .

The measured results are shown in Fig.5, where $230 \mathrm{MHz}$ of bandwidth achieved slightly wider with respect to simulation and an insertion loss of $2.27 \mathrm{~dB}$ is obtained at the frequency center $4 \mathrm{GHz}$ compared to $0.89 \mathrm{~dB}$ in simulation . The rejection out of band on both side of the transmission band is over $20 \mathrm{~dB}$, which guarantee a good selectivity and avoid interference with connected other planar microwave devices. In the same line, a good superposition in the transmission and reflexion coefficient is achieved between the measurement and simulations results. The bandwidth in measurements is slightly wider than simulation .This can be explained by the effect of soldering SMA port to transitions. While the matching is over $15 \mathrm{~dB}$ for both measurements and simulations.

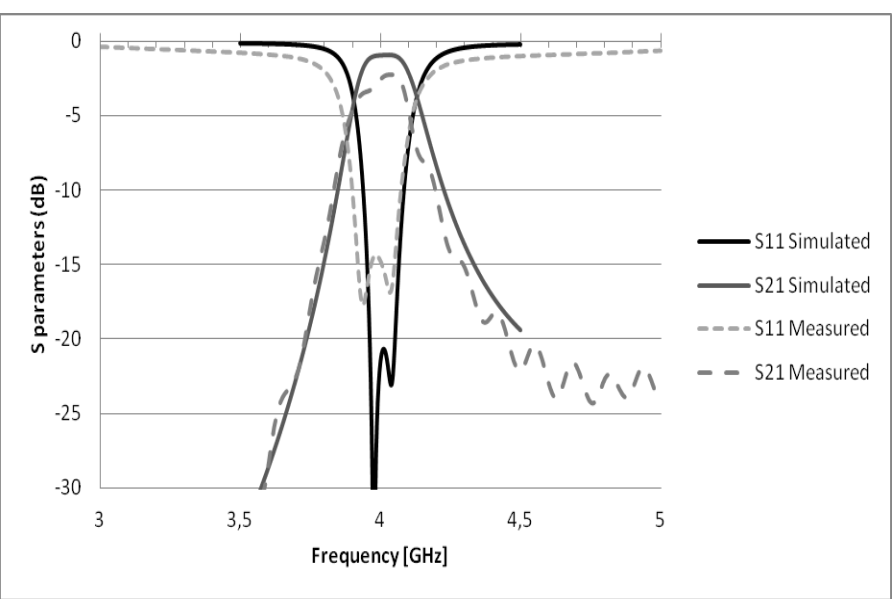

Fig. 5. Simulated and measured scattering parameters of the SIW filter on TLX 9 conventional substrate

\section{B. SIW Band pass filter physical etching technique and paper substrate}

The same design of the two cavities of the filter is employed as above. The dimensions are optimized for bandpass operation centered at $4 \mathrm{GHz}$. This prototype is designed to test the accuracy and the precision of the milling machine in the fabrication of devices based on paper substrate and aluminum metallization.

Transitions from the $50 \mathrm{Ohm}$ feeding microstrip line to the filter are designed to achieve good performance in terms of matching and insertion loss [9]. The design and the dimensions of this component are displayed in Fig. 6.

Both top and bottom faces of the fabricated filter are shown in Fig. 7, where the bottom layer is a fully metalized ground plane. The photograph shows the precision and the accuracy of the proposed technique and machine in sharp corners of the transitions also with this kind of substrate.

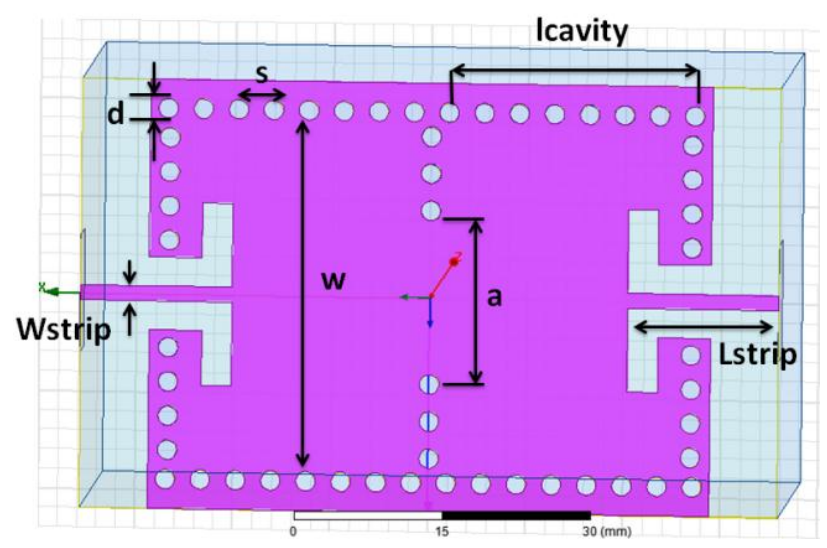

Fig. 6. Geometry of the SIW filter on paper (Lstrip $=15.5 \mathrm{~mm}$, Wstrip $=1.52$ $\mathrm{mm}, a=16.8 \mathrm{~mm}$, lcavity $=26.8 \mathrm{~mm}, \mathrm{w}=38 \mathrm{~mm}, d=2 \mathrm{~mm}, s=3.6 \mathrm{~mm}$ ) 


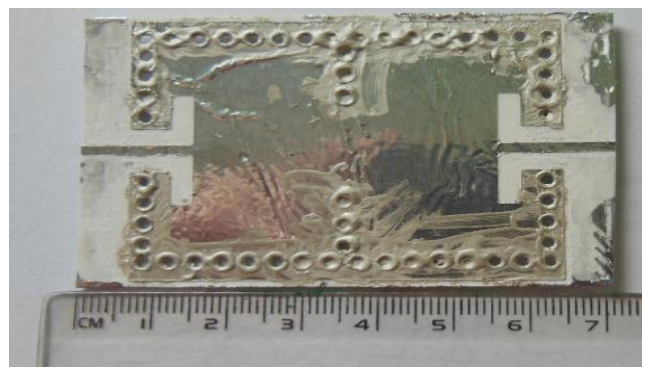

(a)

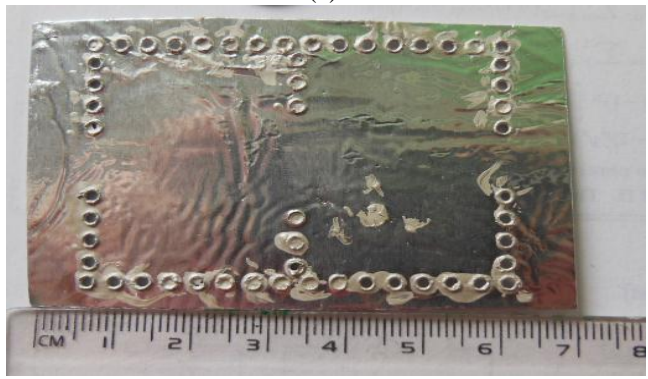

(b)

Fig. 7. Paper-based SIW filter (a) top face (b) bottom face

The simulated and measured frequency responses of the component are reported to Fig.8. An insertion loss of $5 \mathrm{~dB}$ at $4 \mathrm{GHz}$ is obtained. The measured frequency response presents a shift in low frequency with respect to the simulated results. This discrepancy could be caused by small inaccuracies during the characterization of the electrical parameters of the paper substrate, which is a very critical step. In addition, the performance of the proposed filter may be affected by the non perfect metallization of the vias as discussed above.

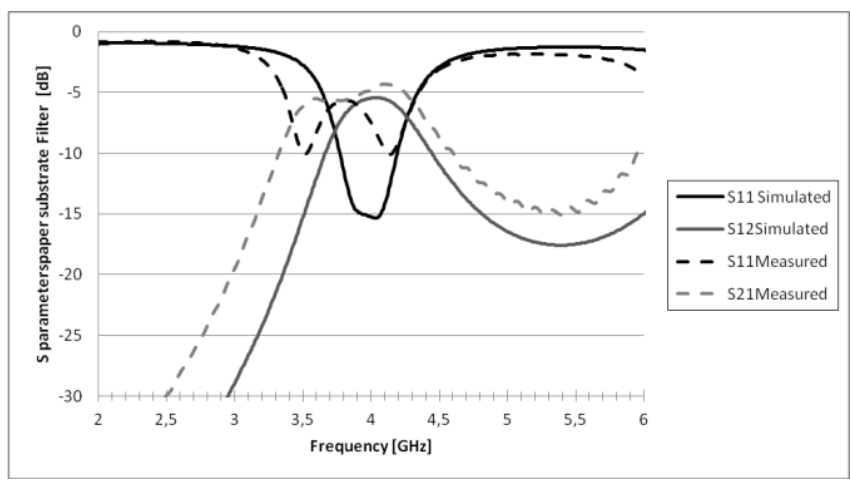

Fig. 8. Simulated and measured scattering parameters of the SIW filter on paper

As shown in Fig. 8 , the measured bandwidth $880 \mathrm{MHz}$ achieved is four times wider than with the conventional Taconic substrate mentioned above. In fact, the resonance of the two cavities are merged to enlarge the bandwidth. The out of band rejection is over $15 \mathrm{~dB}$ in measurement on lower and upper side of the transmission band and validating the simulation results which maintain the target specifications in terms of minimum attenuation required to avoid interference. Moreover, the reflexion coefficient shows the two similar poles in the transmission band around the central frequency. A vector network analyzer (VNA) is employed to measure the frequency response of the prototyped filter.
There is an experimental difficulty to solder a standard SMA connector on aluminum transition for measurements . Therefore, the filter is measured with a universal test fixtures designed for the tests of paper components. In fact, it contains fixed and movable pin connectors to accommodate different length of substrate Fig. 9.

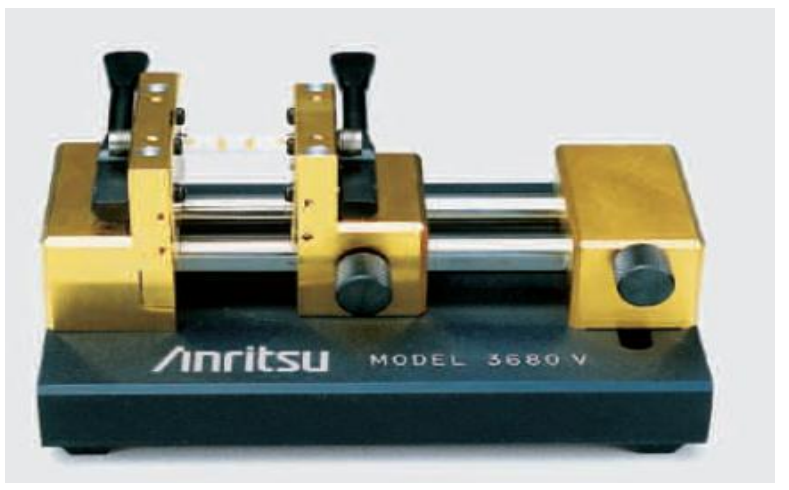

Fig. 9. Anritsu Universal test fixtures facilitating measurement of structures based paper

\section{CONCLUSION}

This work highlights for the first time a comparison between standard substrate used with (PCB ) technology and new physical etching technology. The development of green electronic fabrication components is attracting many researchers as well as industrial to avoid chemical impact on the environment. The bandwidth of filter based paper substrate is almost four time wider than conventional substrate TLX . Based on the results achieved in this paper, this implementation can be adopted for the realization of devices for the next conformal and wireless systems since it combines the advantage of low cost, flexible, eco-friendly materials and simple process of fabrication. Further studies and tests are in progress to improve the paper losses. In the same time, some steps in the manufacturing process are identified to pave the way for possible future works in Wireless sensor network and internet of things applications .

\section{ACKNOWLEDGMENT}

The authors wish to express their gratitude to Prof $\mathrm{M}$. Bozzi and his team lab, the Dept. Electrical, Computer and Biomedical Engineering, University of Pavia, for their technical assistance for fabricating the experimental prototypes.

\section{REFERENCES}

[1] R. Moro, S. Kim, M. Bozzi, and M. Tentzeris, "Novel Inkjet-Printed Substrate Integrated Waveguide (SIW) Structures on Low-Cost Materials for Wearable Applications," 42th European Microwave Conference 2012 (EuMC 2012), Amsterdam, The Netherlands, Oct. 28Nov. 2, 2012.

[2] G. Orecchini, V. Palazzari, A. Rida, F. Alimenti, M. M. Tentzeris, and L. Roselli, "Design and fabrication of ultra-low cost radio frequency identification antennas and tags exploiting paper substrates and inkjet printing technology," IET Microw. Antennas Propag., vol. 5, no. 8, pp.993-1001, Jul. 2011.

[3] R. Moro, S. Agneessens, H. Rogier, and M. Bozzi, "Wearable Textile Antenna in Substrate Integrated Waveguide Technology," IET Electronics Letters, Vol. 48, No. 16,pp. 985-987, August 2, 2012. 
[4] R. Moro, A. Collado, S. Via, A. Georgiadis, and M. Bozzi, "Plasticbased Substrate Integrated Waveguide (SIW) Components and Antennas," 42th European Microwave Conference 2012 (EuMC 2012), Amsterdam, The Netherlands, Oct. 28-Nov. 2, 2012.

[5] F.Alimenti, P.Mezzanotte, M.Dionigi, M.Virili and L.Roselli, "Microwave Circuits in Paper Substrates Exploiting Conductive Adhesive Tapes ," IEEE Microwave and Wireless Components Letters, Vol. 22, No. 12, December 2012.

[6] R. Garg, I. Bahl, M. Bozzi, Microstrip Lines and Slotlines, $3^{\text {rd }}$ ed. Norwood, MA, USA: Artech House, 2013.

[7] K. Wu, D. Deslandes and Y. Cassivi, "The Substrate Integrated Circuits - A New Concept for High-Frequency Electronics and Optoelectronics, "TELSKIS 2003, Nis, Serbia and Montenegro, pp. Oct. 2003.

[8] J. E. Rayas-Sanchez and V. Gutierrez-Ayala, "A General EM-Based Design Procedure for Single-Layer Substrate Integrated Waveguide Interconnects with Microstrip Transitions", IEEE MTT-S Int. Microwave Symp. Dig., Atlanta, GA, Jun. 2008, pp. 983-986.

[9] X.Chen, K. Wu, D. Drolet, "Substrate Integrated Waveguide Filter with Improved Stopband Performance for Satellite Ground Terminal," Microwave Theory and Techniques, IEEE Transactions on Vol 57 ,pp.674-683, 2009.

[10] M. Bozzi, A. Georgiadis, and K. Wu, "Review of Substrate Integrated Waveguide (SIW) Circuits and Antennas," IET Microwave Antennas and Propagation, Vol. 5, No. 8, pp. 909- 920, June 2011.

[11] F. Giuppi, A. Collado, A. Georgiadis, and M. Bozzi, "A Compact, Single-Layer Substrate Integrated Waveguide (SIW) Cavity-Backed Active Antenna Oscillator," IEEE Antennas and Wireless Propagation Letters, Vol. 11, pp. 431-433, 2012.
[12] H.Abdelali, R.Bedira, H.Trabelsi, A.Gharsallah, S.Moscato, R. Moro, M. Pasian, M. Bozzi and L. Perregrini, "Innovative Technique for Substrate Integrated Waveguide Implementation on Paper Substrate," 14th Mediterranean Microwave Symposium (MMS 2014), Marrakech, Morocco, Dec 12-14,2014.

[13] H.Abdelali, R.Bedira, H.Trabelsi and A.Gharsallah, "Development of Substrate Integrated Waveguide BPF for Wireless Sensor Network Applications," International Journal of Innovative Engineering and Emerging Technology (IJIEET), Vol. 2, Issue 2, May-June 2016.

[14] Q.Wang and J.Bornemann, " Triple-Pass-Band, Dual-Stop-Band UWB Antenna With Substrate integrated Waveguide Resonators," Microwave and optical Technology Letters, Vol. 56, No. 6, June 2014.

[15] S.Moscato, R.Moro, M.Pasian, M.Bozzi and L.Perregrini, "Innovative manufacturing approach for paper-based substrate integrated waveguide components and antennas," IET Microw. Antennas Propag., 2016, Vol. 10, Iss. 3, pp. 256-263.

[16] M.Bozzi, L.Perregrini, and K. Wu, "Modeling of conductor, dielectric and radiation losses in substrate integrated waveguide by the boundary integral-resonant mode expansion method " IEEE Trans. Microw. Theory Tech., 2008, 56, (12), pp. 3153-3161.

[17] M.Bozzi, M.Pasian.,L.Perregrini and al., " On the losses in substrate integratedwaveguides and cavities ," Int. J. Microw. Wirel. Technol., 2009, 1, (5), pp. 395-401.

[18] R. J , Cameron., C.M Kudsia. and R.R., Mansour, “ Microwave filters for communication systems: fundamentals, design and applications," (John Wiley and Sons, Inc., New Jersey, 2007). 